\title{
Clinical features and outcomes in patients with non-acoustic cerebellopontine angle tumours
}

\author{
Connor L Mallucci, Victoria Ward, A Simon Carney, Gerard M O’Donoghue, \\ Iain Robertson
}

\begin{abstract}
Objectives-Non-acoustic tumours of the cerebellopontine angle differ from vestibular schwannomas in their prevalence, clinical features, operative management, and surgical outcome. These features were studied in patients presenting to the regional neuro-otological unit.

Methods-A retrospective analysis of clinical notes identified 42 patients with non-acoustic tumours of the cerebellopontine angle. Data were extracted regarding presenting clinical features, histopathological data after surgical resection, surgical morbidity and mortality, and clinical outcome (mean 32 months follow up).

Results-The study group comprised 25 meningiomas $(60 \%), 12$ epidermoid cysts/ cholesteatomata (28\%), and five other tumours. In patients with meningiomas, symptoms differed considerably from patients presenting with vestibular schwannomas. Cerebellar signs were present in $52 \%$ and hearing loss in only $68 \%$. Twenty per cent of patients had hydrocephalus at the time of diagnosis. After surgical resection, normal facial nerve function was preserved in $75 \%$ of cases. In the epidermoid group, fifth, seventh, and eighth nerve deficits were present in $42 \%, 33 \%$, and $66 \%$ respectively. There were no new postoperative facial palsies. There were two recurrences $(17 \%)$ requiring reoperation. Overall, there were two perioperative deaths from pneumonia and meningitis.

Conclusions-Patients with non-acoustic lesions of the cerebellopontine angle often present with different symptoms and signs from those found in patients with schwannomas. Hearing loss is less prevalent and cerebellar signs and facial paresis are more common as presenting features. Hydrocephalus is often present in patients presenting with cerebellopontine angle meningiomas. Non-acoustic tumours can usually be resected with facial nerve preservation.

(F Neurol Neurosurg Psychiatry 1999;66:768-771)
\end{abstract}

Keywords: cerebellopontine angle; brain tumour; meningioma

Tumours of the cerebellopontine angle account for $8 \%-10 \%$ of all intracranial tumours. Acoustic neuromas (vestibular schwannomas), arising from the neurilemmal junction of the vestibular nerve, account for between $80 \%-$
$90 \%$ of these tumours. ${ }^{1-4}$ Other sources of tumour in this region include epidermal cell rests (giving rise to epidermoid cysts, dermoid cysts, and cholesteatomata), arachnoid villi/ granulations and the meninges (meningiomas, arachnoid cysts), primary intrinsic lesions (gliomas, ependymomas), fat cells (lipomas), tumours extending from the cranial base (for example, glomus jugulare tumours), vascular lesions (haemangiopericytomas), and secondary tumours. ${ }^{12}$

The relation of each of these tumours to the bone of the cranial base varies greatly and has implications for surgical management. Understanding the precise relation of each tumour to the adjacent neural, vascular, and sensory structures is critically important if the best functional outcome is to be achieved after surgical resection. ${ }^{1}$ Accurate preoperative diagnosis of tumour type is therefore of the utmost importance in planning the optimal management strategy for each patient..$^{5-8}$

In addition, accurate diagnosis enables informed discussion with patients about the natural history of the untreated disease compared with the potential risks of surgical and non-surgical management.

The purpose of this study was to review the pattern of presentation of a consecutive series of non-acoustic tumours of the cerebellopontine angle, to assess the ability to predict the histological diagnosis preoperatively, and to analyse the outcome of treatment.

Patients and methods

Patients were identified from a continually updated database of all cerebellopontine angle tumours presenting to the combined otoneurosurgical regional unit in Nottingham. Data were recorded retrospectively from clinical notes and radiological imaging including CT and MRI (the second including both conventional T1 and T2 images with and without contrast). More recently, MRI with constructive interference in the steady state ${ }^{9}$ (CISS) images has also been available.

The following data were recorded: age, sex, histological review, duration of symptoms, clinical symptoms and signs, preoperative and postoperative radiological investigations, surgical approach, complications, and subsequent progress.

Results

Forty two patients were identified who had been treated by this department between 1980 and 1996. There were seven men and 35 


\begin{tabular}{lll}
\hline & Meningiomas & $\begin{array}{l}\text { Epidermoids/ } \\
\text { cholesteatomata }\end{array}$ \\
\hline Symptom duration (median in months) & 12 & 24 \\
Hydrocephalus & $20 \%$ & $8 \%$ \\
Cerebellar symptoms & $52 \%$ & $33 \%$ \\
Hearing loss (subjective) & $32 \%$ & $50 \%$ \\
Hearing loss (objective) & $68 \%$ & $66 \%$ \\
Preoperative fifth nerve symptoms & $36 \%$ & $42 \%$ \\
Preoperative seventh nerve symptoms & $8 \%$ & $33 \%$ \\
Postoperative seventh nerve palsy (permanent) & $2(10 \%)$ & 0 \\
Postoperative seventh nerve palsy (partial recovery) & $3(15 \%)$ & 0 \\
Postoperative absent gag/ swallow & $4(20 \%)$ & $2(25 \%)$ \\
Haematoma & 1 & 0 \\
Infection & 1 & 0
\end{tabular}

women, with a median age of 54 years. Median follow up postsurgery was 3 years (range 8 months-14 years).

Patients with meningiomas $(n=25)$ comprised most of the group, followed by patients with epidermoid cysts/cholesteatomata $(n=12)$. Other lesions included two haemangiopericytomas, a metastatic adenocarcinoma, and an ependymoma.

\section{MENINGIOMAS}

In our series, there were 25 patients with meningiomas, 21 women and four men with a median age of 55 years. Median duration of symptoms was 12 months. About half the patients had signs of cerebellar ataxia at presentation (table), with only a third presenting with subjective hearing loss. Two patients presented with facial pain, three with facial numbness, and four with symptoms of raised intracranial pressure. Only three patients had tinnitus on direct questioning, but tinnitus was not a major presenting symptom. On pure tone audiometry, $68 \%$ had evidence of asymmetric $(>10 \mathrm{~dB}$ four tone average) sensorineural hearing impairment.

Radiological work up included CT in 21 and MRI in 16 patients. In all 20 patients operated on with available histology and one in whom pathology was obtained postmortem, the radiological reports predicted a likely diagnosis of meningioma in all 21 patients giving 100\% predictive accuracy. Five patients $(20 \%)$, had hydrocephalus at presentation.

\section{Surgical management of meningiomas}

Twenty patients went on to have surgical resections of which $13(65 \%)$ were complete and seven $(35 \%)$ were subtotal. The surgical approach was retrosigmoid in all but one patient, in whom a translabyrinthine approach was used. Two patients who were elderly had ventriculoperitoneal shunts only. Two patients with small tumours are being followed up with regular periodical scanning and the remaining patient presented in extremis with hydrocephalus and coma and died before any surgical intervention.

\section{Complications of cerebellopontine angle meningioma surgery}

Complications are shown in the table. In $75 \%$ the facial nerve was spared, in three patients there was partial recovery at follow up, reaching House and Brackman ${ }^{10}$ grades 1 or 2 and in only two patients were permanent unre- coverable (grade 3 or more) facial palsies recorded. Four patients had their lower cranial nerves (ninth and 10th) affected with diminished gag reflex and/or swallow. In one patient a long term tracheostomy was required. Other complications included third and 12th nerve palsies which recovered spontaneously, a postoperative haematoma that required emergency evacuation, and four wound CSF leaks which stopped spontaneously or with only a further suture on the ward.

There were two postoperative deaths. Both died in the month after surgery. The first patient, requiring the emergency evacuation of postoperative haematoma, died of aspiration pneumonia partially attributable to poor gag and swallow reflexes secondary to ninth/10th nerve palsies after surgery. The second patient succumbed to postoperative infection and ventriculitis and died from systemic sepsis.

\section{Follow up}

There have been no recurrences in patients with planned complete resections and in those with known subtotal resections, the residuum is being followed up with regular imaging but there have been no episodes of tumour progression requiring reoperation to date.

EPIDERMOID CYSTS AND CHOLESTEATOMATA

There were 12 patients with epidermoid cysts/ cholesteatomata, together comprising $29 \%$ of the total group. The median age was 42 years with a female:male ratio of 5:1. Median duration of symptoms was 24 months (table). Symptomatic hearing loss as a presenting symptom was present in $50 \%$ of patients $(66 \%$ had diminished hearing on formal testing). Thirty two per cent and $42 \%$ of patients respectively presented with facial nerve and trigeminal nerve involvement. Other presenting symptoms and signs included four patients with cerebellar signs, one with dysphagia and one with diplopia. Only one patient had hydrocephalus and again none had tinnitus as a main presenting symptom.

Radiological investigation included $10 \mathrm{CT}$ scans and nine MRI scans. The suggested radiological diagnosis in this group compared with histological diagnosis was $88 \%$ accurate.

\section{Surgery}

Eight of the patients went on to have surgical resections of which five were complete excisions and three were subtotal. Four patients with small tumours are being followed up prospectively with interval scanning. The approach was retrosigmoid in six and translabyrinthine in two patients.

\section{Complications}

There have been no new facial palsies; two patients had a diminished gag reflex postoperatively although neither have required tracheostomy. There was one CSF leak, which was successfully managed with further suturing to the wound. There were no infective complications and no mortality in this group of patients. Postoperative hearing was unchanged $(<10 \mathrm{~dB}$ 
4 tone average) in all patients undergoing nondestructive approaches.

Follow up

With a median follow up of 58 months there have been two recurrences in the operated group, both patients in whom it was known that subtotal excisions had been performed. Both required reoperation due to the symptomatic regrowth of residual tumour.

The four patients being treated conservatively show no tumour progression to date.

\section{Miscellaneous tumours}

These included two haemangiopericytomas, one metastatic adenocarcinoma, one ependymoma, and one lesion of unknown histology. Four of these have had surgery, with no recurrences in the two patients with haemangiopericytomas. Both patients with ependymoma and adenocarcinoma have since re-presented with metastases at extracranial sites.

\section{Discussion}

Acoustic neuromas remain by far the most prevalent lesion to be found in the cerebellopontine angle. ${ }^{4}$ Meningiomas are the second most common tumour to be found in this site, accounting for exactly $6.5 \%$ of all cases in two separate British series. ${ }^{211}$ Meningiomas accounted for $60 \%$ of our series, more than the $33 \%$ and $32 \%$ reported respectively in the London $^{11}$ and Cambridge ${ }^{2}$ series. These differences may reflect local referral practices. Epidermoid cysts and cholesteatomata are often included together in reported series and if we combine numbers of the two patient groups, they represent $29 \%$ of total numbers, more in keeping with the proportion found in other series. ${ }^{12}$

The clinical features of haemangiopericytomas, metastases, and other rare lesions of the cerebellopontine angle have been well documented by other workers, ${ }^{12-18}$ and their rarity does not allow meaningful comparisons to be made about prevalence rates.

In this study, as in previous reports, nonacoustic tumours of the cerebellopontine angle are more likely to present with cerebellar symptoms than schwannomas. ${ }^{213}$ The most common complaint was of unsteadiness or falling persistently to one side and was accompanied by clinically apparent ataxia. However, other symptoms associated with cerebellar dysfunction (for example, intention tremor and nystagmus) were uncommon findings. The latter infrequency in our series is a marked difference from other workers' findings with similar numbers of patients presenting with ataxia. ${ }^{3}$ It has been reported that these symptoms are often considered mild ${ }^{11}$ and this would seem to be borne out by our study. Those patients who presented primarily with cerebellar signs experienced symptoms for a lengthy period (longer than the mean) before diagnosis.

Cranial nerve palsies were the main source of prediagnosis morbidity with sensorineural hearing loss being the most common presenting symptom and sign. However, compared with acoustic neuroma series hearing loss is much less prevalent in keeping with other experiences. ${ }^{18}{ }^{19}$ Formal preoperative and postoperative assessment of hearing in this group is essential and will not only have a bearing on the choice of surgical approach adopted but will help clinicians to optimise resection techniques to maximise the chance of some hearing preservation.

Trigeminal nerve symptoms are more prominent in non-acoustic lesions ${ }^{18}$ and particularly with epidermoid cysts, are more likely to cause trigeminal pain than altered sensation. ${ }^{20}$ This is one symptom that can be gratifyingly improved by surgery.

We were surprised by the relative infrequency of reported tinnitus, $(12 \%)$ which has generally been found in association with hearing loss in other series ${ }^{211}{ }^{19}$ although others have also pointed out that it is also an infrequent complaint in non-acoustic cerebellopontine angle lesions. ${ }^{1}$ We have found no cases of true rotatory vertigo such as have been reported by other workers. ${ }^{11} 19$

Overall symptomatology is still dependent on tumour size and the exact location of the lesion and we agree with others that there are not enough clinical differences in presentation to allow preoperative differentiation from acoustic tumours on history and examination alone. ${ }^{12}$ There are, however, often relevant clinical clues that should alert the clinician that the lesion may well be non-acoustic ${ }^{2}$ and this may be of importance if the radiological findings are inconclusive.

Various approaches have been shown to be feasible in resecting tumours in the posterior fossa and have been well reviewed. ${ }^{21}$ Most of our cases were exposed through a retrosigmoid approach. This has afforded good visualisation and allowed resection of the tumour without recourse to excessive retraction. However, for the petroclival meningiomas a transpetrosal approach has recently proved to us to have major advantages. In the patient with no prospect of useful postoperative hearing, a translabyrinthine approach may be appropriate especially for the tumours involving the internal auditory meatus ${ }^{11}$ although it must always be remembered that hearing may occasionally improve or even return to normal after the resection of non-acoustic cerebellopontine angle tumours ${ }^{22}{ }^{23}$ and destructive approaches must therefore be used selectively.

Despite using an approach that offers optimal exposure, certain clival meningiomas and extensive epidermoids are difficult to remove in their entirety without subjecting the patient to unacceptable morbidity. ${ }^{1}$ Other studies have also included patients with incomplete excisions. ${ }^{18}$ In the case of an extensive epidermoid cyst, the tumour may have extensive adherent capsule extensions folding around important structures. ${ }^{1}$ Clival meningiomas may be adherent to the brain stem or may envelop arteries or nerves which need to be preserved. Our study reinforces the view of others ${ }^{1}$ that, despite a relatively short follow up, due to the biology of these tumours, incomplete excision can still afford good lifestyle with minimal morbidity for years with 
no essential requirement for further surgery. If repeat surgery is required, it may be at very infrequent intervals, often as long as a decade apart. ${ }^{1}$

Because of the implications for surgical management, it is essential that non-acoustic tumours of the cerebellopontine angle are correctly diagnosed preoperatively. ${ }^{2}$ Fortunately, with the availability of high quality MRI, an accurate diagnosis can be made in most cases ${ }^{1}$ although in one recent series, $25 \%$ of cerebellopontine angle meningiomas were radiologically thought to be vestibular schwannomas and the exact nature of atypical tumours was only demonstrated in $67 \%$ of cases. ${ }^{8}$ Computed tomography does not always disclose the nature of an atypical cerebellopontine angle lesion ${ }^{20}$ and there is no doubt that MRI is now the radiological investigation of choice. ${ }^{12}$ Even with contrast enhanced MRI, small lesions may still be radiologically similar to schwannomas ${ }^{24}$ and complex new imaging techniques such as CISS may be necessary to show the true nature and anatomy of a non-acoustic lesion. ${ }^{9}$ We would recommend the use of CISS MRI sequences when there is any doubt as to the exact nature of a cerebellopontine angle mass. A detailed discussion on the radiological diagnosis of non-acoustic tumours is beyond the scope of this paper and has been well covered by other authors. ${ }^{12} 25-28$

Whereas vestibular schwannomas rarely recur after complete resection, this is not the case for other cerebellopontine angle lesions. ${ }^{8}$ Ten to $13 \%$ of patients with cerebellopontine angle meningiomas develop recurrent disease, thought to be due to inadequate bone removal at the time of initial surgery or the persistence of tumour within the dura. ${ }^{811}$ Although some series report good results after the resection of epidermoid cysts/cholesteatomata, ${ }^{8}$ other workers have found that they are notoriously prone to recurrence ${ }^{29} 30$ and long term follow up is essential. Rarer lesions have an even higher rate of recurrence and regular follow up and rescanning of patients with these rare tumours is essential. ${ }^{2}$

\section{Conclusion}

Non-acoustic tumours of the cerebellopontine angle often present with a different range of symptoms and signs to those found in patients with schwannomas. Hearing loss is less prevalent and cerebellar signs and facial paresis are more common as presenting features. Hydrocephalus is often present in patients presenting with cerebellopontine angle meningiomas.

All non-cerebellopontine angle lesions are more prone to recurrence than acoustic neuromas and long term follow up is recommended. Useful hearing can be preserved in a high proportion of cases and these rare tumours can usually be resected with preservation of facial nerve function.
1 Lalwani AK. Meningiomas, epidermoids, and other nonacoustic tumors of the cerebellopontine angle. Otolaryngol Clin North Am 1992:25:707-28.

2 Moffat DA, Saunders JE, McElveen JT Jr, et al. Unusual cerebello-pontine angle tumours. F Laryngol Otol 1993;107: 1087-98.

3 Tekkok IH, Suzer T, Erbengi A. Non-acoustic tumors of the cerebellopontine angle. Neurosurg Rev 1992;15:117-23.

4 Brackmann DE, Kwartler JA. A review of acoustic tumors: 1983-8. Am F Otol 1990;11:216-32.

5 Tator CH, Duncan EG, Charles D. Comparisons of the clinical and radiological features and surgical management of posterior fossa meningiomas and acoustic neuromas. Can F Neurol Sci 1990;17:170-6.

6 Prasad S, Kamerer DB, Hirsch BE, et al. Preservation of vestibular nerves in surgery of the cerebellopontine angle: effect on hearing and balance function. Am $\mathcal{f}$ Otolaryngol 1993;14:15-20.

7 Nedzelski J, Tator C. Other cerebellopontine angle (non8 Grey PL, Moffat DA, Hardy DG. Surgical results in unusual Grey PL, Moffat DA, Hardy DG. Surgical results in unusual
cerebellopontine angle tumours. Clin Otolaryngol 1996;21: cerebellop $237-43$.

9 Casselman JW, Kuhweide R, Deimling M, et al. Constructive interference in steady state-3DFT MR imaging of the inner ear and cerebellopontine angle. Am $\mathcal{f}$ Neuroradiol 1993;14:47-57.

10 House JW, Brackmann DE. Facial nerve grading system. Otolaryngol Head Neck Surg 1985;93:146-7.

11 Thomas NW, King TT. Meningiomas of the cerebellopontine angle. A report of 41 cases. $\mathrm{Br} f$ Neurosurg 1996;10:59-68.

12 Anderson C, Krutchkoff D, Ludwig M. Carcinoma of the lower lip with perineural extension to the middle cranial fossa. Oral Surgery Oral Medicine Oral Pathology 1990;69: fossa. Oral $614-18$.

13 Brackmann DE, Bartels LJ. Rare tumors of the cerebellopontine angle. Otolaryngol Head Neck Surg 1980;88:555-9.

14 Cohen DL, Diengdoh JV, Thomas DG, et al. An intracranial metastasis from a PRL secreting pituitary tumour. Clin Endocrinol (Oxf) 1983;18:259-64.

15 Kohan D, Downey LL, Lim J, et al. Uncommon lesions presenting as tumors of the internal auditory canal and cerebellopontine angle. Am f Otol 1997;18:386-92.

16 McMenomey SO, Glasscock ME 3rd, Minor LB, et al. Facial nerve neuromas presenting as acoustic tumors. Am $\mathcal{F}$ Otol 1994;15:307-12.

17 Minami M, Hanakita J, Suwa H, et al. Solitary metastasis of lung cancer to the cerebellopontine angle: case report. Neurol Med Chir (Tokyo) 1996;36:172-4.

18 Molnar P, Nemes Z. Hemangiopericytoma of the cerebellopontine angle. Diagnostic pitfalls and the diagnostic value of the subunit A of factor XIII as a tumor marker. Clin Neuropathol 1995;14:19-24.

19 Baguley DM, Benyon GJ, Grey PL, et al. Audio-vestibular findings in meningioma of the cerebello-pontine angle: a retrospective review. F Layngol Otol 1997;111:1022-6.

20 Fitt AW, Pigott TJ, Marks PV. Undiagnosed epidermoid cyst presenting as trigeminal neuralgia: a need for MRI. $B r f$ Neurosurg 1994;8:101-3

21 Yasargil MG, Morarara RW, Cicur CM. Meningiomas of the basal posterior fossa. In: Krayenbuhl H, ed. Advances and technical standards in neurosurgery. New York: SpringerVerlag, 1980:3-115.

22 Vellutini EA, Cruz OL, Velasco OP, et al. Reversible hearing loss from cerebellopontine angle tumors. Neurosurgery 1991;28:310-2.

23 Cane MA, Lutman ME, O’Donoghue GM. Transiently evoked otoacoustic emissions in patients with cerebellopontine angle tumors. Am F Otol 1994;15:207-16.

24 Bassi P, Piazza P, Cusmano F, et al. MR cisternography of the cerebello-pontine angle and internal auditory canal in diagnosis of intracanalicular acoustic neuroma. Neuroradiology 1990;31:486-91.

25 Helie O, Soulie D, Sarrazin JL, et al. Magnetic resonance imaging and meningiomas of the posterior cerebral fossa: 31 cases. $\mathcal{F}$ Neuroradiol 1995;22:252-70.

26 Lalwani AK, Jackler RK. Preoperative differentiation between meningioma of the cerebellopontine angle and acoustic neuroma using MRI. Otolaryngol Head Neck Surg 1993;109:88-95.

27 Mawhinney RR, Buckley JH, Worthington BS. Magnetic resonance imaging of the cerebello-pontine angle. Br $\mathcal{F}$ Radiol 1986;59:961-9.

28 Mafee MF, Kumar A, Heffner DK. Epidermoid cyst (cholesteatoma) and cholesterol granuloma of the temporal bone and epidermoid cysts affecting the brain. Neuroimaging Clin N Am 1994;4:561-78

29 De Micheli E, Bricolo A. The long history of a cerebello-pontine angle epidermoid tumour: a case report and lessons learned. Acta Neurochir (Wien) 1996;138:350 4.

30 Parikh S, Milosevic M, Wong CS, et al. Recurrent intracranial epidermoid cyst treated with radiotherapy. F Neurooncol 1995;24:293-7. 